\title{
A general solution for the receding contact problem of a functionally graded layer resting on a Winkler foundation
}

\author{
G. Adiyaman*, A. Birinci \\ Karadeniz Technical University, Civil Engineering Department, 61080, Trabzon, Turkey
}

\begin{abstract}
In this paper, the receding contact problem of functionally graded (FG) layer resting on a Winkler foundation is considered. It is assumed that the shear modulus of the layer change functionally along the depth whereas Poisson ratio remains constant. Arbitrary concentrated loads by means of arbitrary rigid punches are applied to the top of the layer. The problem is considered as a plain strain problem. A general formulation is obtained using elasticity theory and Fourier integral transform. Obtained formulation is valid for both symmetric and asymmetric systems. A parametric study is carried out to investigate the effect of material properties and loading on contact distances and contact pressures. It is found that, increasing rigidity of the bottom of the FG layer compared to the top of the FG layer, the contact distances between the circular punch and FG layer contact surface decreases whereas maximum contact pressure increases. In addition, placement of the rigid punches has an effect on the contact distances and contact pressures.
\end{abstract}

\section{Keywords}

Receding contact, Elasticity; Functionally graded material; Fourier transform; Receding contact

Received: 17 August 2018; Accepted: 06 September 2018

ISSN: 2630-5763 (online) C 2018 Golden Light Publishing® All rights reserved.

\section{Introduction}

There are many engineering applications where the stress analysis at the interface between two bodies in contact is principal in the structural design as the response of the structure depends on it. Examples of these applications in engineering are railways, foundation grillages, connecting rods, joint and support elements, rolling mills, pavements of highway and airfield etc. [1,2]. So, problems involving the contact of two separate bodies pressed against each other have been widely studied by many researchers. Although the contact area increases after the application of the load in many cases, there are others where the contact area becomes smaller. This kind of problem is called receding in literature.

Among the analytical studies on receding contact, the followings are recorded in literature. Keer et.al. [3] solved the smooth receding contact problem between an elastic layer and a half space when two bodies were pressed against each other by considering both plane and axisymmetric cases. The frictionless contact problem for an elastic layer resting on two quarter planes and loaded compressively was solved by Erdogan and Ratwani [4]. Civelek and Erdogan [5] investigated the general axisymmetric double frictionless contact problem for an elastic layer resting on a half space and pressed by an elastic stamp. The smooth receding contact problem for an elastic layer

\footnotetext{
Corresponding author

E-mail: gadiyaman@ktu.edu.tr
} 
pressed against a half space by frictionless semiinfinite elastic was examined by Gecit [9]. Akavc1 [6] studied a contact problem for an elastic layer supported by two elastic quarter planes both symmetrical and axisymmetric loadings. Comez et.al. [7] solved double receding contact problem for two elastic layers having different elastic constants and heights and pressed by a rigid stamp. Kahya et.al. [8] considered a frictionless receding contact problem between an anisotropic elastic layer and an anisotropic elastic half plane, when the two bodies were pressed together by means of a rigid circular stamp. Yaylacı and Birinci [9] studied a receding contact problem of two elastic layers supported by two elastic quarter planes. The solution of a receding contact problem using an analytical method and a finite element method was examined by Oner et.al. [10]. Karabulut et. al. [11] studied a receding contact problem of layer resting on a half plane and pressed by two flat blocks placed symmetrically

The processing techniques related to functionally graded materials (FGMs) and the importance of these processing was considered by Kieback et. al. [12]. Jin and Batra [13] studied the thermal stresses and the stress intensity factor in an edge-cracked strip of a functionally graded material (FGM) subjected to sudden cooling at the cracked surface. The geometrically nonlinear response of inhomogeneous isotropic and functionally graded plates and shells was considered by Hui-Shen [14]. Sofiyev [15] focused on the thermal buckling of FGM shells resting on a two-parameter elastic foundation. The buckling of cylindrical shells made of FGM in contact with the Pasternak elastic foundation subjected to uniform temperature rise is investigated by Bagherizadeh et. al. [16]. Tornabene [17] investigated recovery of throughthe-thickness transverse normal and shear strains and stresses in statically deformed functionally graded (FG) doubly-curved sandwich shell structures and shells of revolution using the generalized zigzag displacement field and the Carrera Unified Formulation.

A receding contact plane problem for a functionally graded layer pressed against a homogeneous half space was analyzed by El-Borgi et.al. [18]. A multi-layered model for sliding frictional contact analysis of functionally graded materials (FGMs) with arbitrarily varying shear modulus under plane strain-state deformation has been developed by Ke and Wang [19]. The twodimensional frictionless contact problem of a coating structure consisting of a surface coating, a functionally graded layer and a substrate under a rigid cylindrical punch was investigated by Yang and $\mathrm{Ke}$ [20]. Barik et.al. [21] studied the stationary plane contact of a functionally graded heat conducting punch and a rigid insulated half-space. The frictionless contact problem of a functionally graded piezoelectric layered half-plane in-plane strain state under the action of a rigid flat or cylindrical punch was examined by Ke et.al. [22]. Sliding frictional contact between a rigid punch and a laterally graded elastic medium was studied by Dag et.al.[23]. Rhimi et.al. [24,25] considered the axisymmetric problem of a frictionless receding contact between an elastic functionally graded layer and a homogeneous half-space when the two bodies were pressed together and double receding contact between a rigid stamp of axisymmetric profile, an elastic functionally graded layer and a homogeneous half space. Chen and Chen [26] studied the contact behaviors of a graded layer resting on a homogeneous half space and pressed by a rigid stamp. Comez [27] considered a contact problem for a functionally graded layer loaded by means of a rigid stamp and supported by a Winkler foundation. The plane problem of a frictional receding contact formed between an elastic functionally graded layer and a homogeneous half space, when they were pressed against each other, was investigated by El-Borgi et.al. [28]. Adiyaman et. al. [29] studied the receding contact problem of FG layer resting on quarter planes and loaded by a symmetrically places distributed load. The double receding contact problem between a FG layer and a homogeneous layer investigated by $\mathrm{Yan}$ and $\mathrm{Li}$ [30]. Liu et. al. [31] studied the axisymmetric receding contact problem of a FG coating under a rigid circular block. The axisymmetric contact problem of a FG layer resting on an elastic substrate 
investigated by Turan et. al. [32]. Yan and Mi $[33,34]$ studied contact problems of a structure consisted of a FG layer, a homogeneous layer and homogenous half plane.

Although the contact problem of a layer resting on Winkler foundation has been studied [35,27], the problems were solved only for a specific symmetric loading case. However, in this study, a general solution valid for any loading case, whether it is symmetric or asymmetric, is derived. In addition, this solution is compatible for programming purposes. Therefore, a computer program with a graphic user interface is developed and the numerical results are obtained used this program. Obtained numerical results are given in tables and figures.

\section{Definition of the problem}

The general solution of the contact problem of a FG layer of thickness $\mathrm{h}$ resting on a Winkler foundation is considered. It is assumed that the shear modulus of the layer, $G$, changes exponentially thorough the depth as given below whereas the Poisson ratio, $v$, remains constant.

$G=G_{0} e^{\beta y}$

In which, $G_{0}$ is the shear modulus of the layer at the bottom ( $y=0)$ and $\beta$ is the non-homogeneity parameter which represents the change in the shear modulus. The loading and the geometry of the problem is given in Fig. 1 as representatively. The layer is loaded with $n$ concentrated loads by means of arbitrary rigid blocks. It is assumed that the layer is attached to the foundation and the effect of the gravity is neglected. The problem is considered as a plain strain problem.

\section{Formulation of the problem}

The equilibrium equations in terms of displacements (Navier Equations) for a FG layer can be obtained as follows.

$$
\begin{array}{r}
(\kappa+1) \frac{\partial^{2} u}{\partial x^{2}}+(\kappa-1) \frac{\partial^{2} u}{\partial y^{2}}+2 \frac{\partial^{2} v}{\partial x \partial y} \\
+\beta(\kappa-1)+B(\kappa-1) \frac{\partial v}{\partial x}=0 \\
(\kappa-1) \frac{\partial^{2} v}{\partial x^{2}}+(\kappa+1) \frac{\partial^{2} v}{\partial y^{2}}+2 \frac{\partial^{2} u}{\partial x \partial y} \\
+\beta(3-\kappa) \frac{\partial u}{\partial x}+\beta(\kappa+1) \frac{\partial v}{\partial y}=0
\end{array}
$$

in which, $u$ and $v$ are the $\mathrm{x}$ and $\mathrm{y}$ components of the displacement field, respectively; $\sigma_{x}, \sigma_{y}$ and $\tau_{x y}$ are the components of the stress field in the same coordinate system; $\varepsilon_{x}, \varepsilon_{y}$ and $\varepsilon_{x y}$ are the corresponding components of the strain field; and $\kappa$ is a material property defined as $\kappa=3-4 \nu$ for plane strain problems. Eqs. $(3,4)$ are subjected to following boundary conditions.

$$
\begin{aligned}
& \sigma_{y}(x, 0)=-k v(x, 0) \quad-\infty<x<\infty \\
& \tau_{y_{1}}(x, 0)=\tau_{y_{1}}(x, h)=0 \quad-\infty<x<\infty
\end{aligned}
$$

$\sigma_{y_{N}}\left(x, h_{N}\right)=\left\{\begin{array}{cc}-p_{1}(x) & b_{1 L}<x<b_{1 R} \\ \vdots & \vdots \\ -p_{j}(x), & b_{j L}<x<b_{j R} \\ \vdots & \vdots \\ -p_{n}(x) & b_{n L}<x<b_{n R}\end{array}\right.$

$\frac{\partial v_{j}(x, h)}{\partial x}=f_{j} \quad-b_{j L}<x<b_{j R}, j=1,2, \ldots, n$

In these boundary conditions, $k$ is the stiffness parameter of the Winkler foundation, $p_{j}$ ( $j=1,2, \ldots, n)$ represents unknown contact pressures under the $j^{\text {th }}$ block; $b_{j L}$ and $b_{j R}$ are unknown starting and ending points of the contact, respectively; and $f_{j}$ is the derivative of the shape function of the $j^{\text {th }}$ block with respect to $x$. Equilibrium conditions for the problem can be written as follows; 


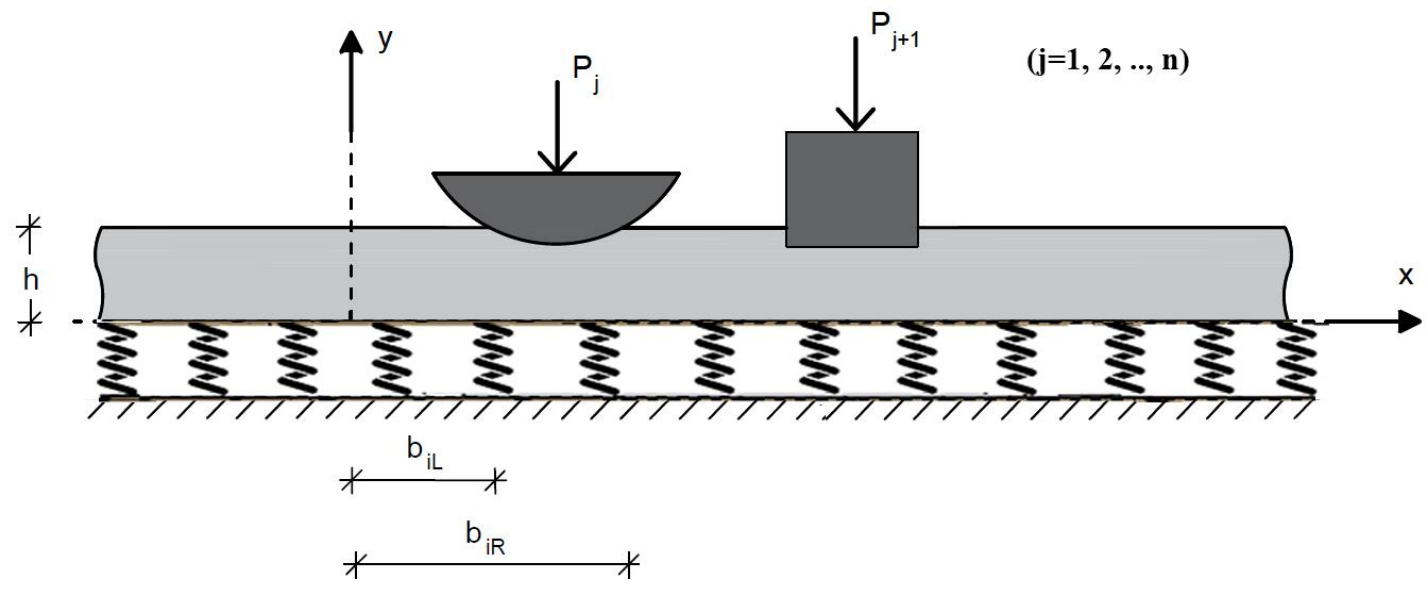

Fig. 1. The geometry and loading of the problem

$\int_{b_{j L}}^{b_{j R}} p_{j}(x) d x=P_{j} \quad(j=1,2, \ldots, n)$

in which, $P_{j} \quad(j=1,2, \ldots, n)$ is the concentrated load applied to top of the $j^{\text {th }}$ block.

\section{Solution of the problem}

Using Fourier integral transform, $u$ and $v$ can be written as follows;

$u(x, y)=\int_{-\infty}^{\infty} \phi(\xi, y) e^{-i \xi x} d x$

$v(x, y)=\int_{-\infty}^{\infty} \psi(\xi, y) e^{-i \xi x} d x$

in which, $\phi(\xi, y)$ and $\psi(\xi, y)$ are the Fourier transform of $u$ and $v$ with respect to the $\mathrm{x}$ coordinate, respectively. Plane elasticity equations $(2,3)$ are partial differential equations and can be converted into ordinary differential equations using Fourier integral transform. The characteristic equation associated with these ordinary differential equations can be obtained as follows.

$$
\begin{aligned}
n_{j}^{4}+2 \beta n_{j}^{3}+ & \left(\beta^{2}-2 \xi^{2}\right) n_{j}^{2}-2 \xi^{2} \beta n_{j} \\
& +\xi^{2}\left(\xi^{2}+\beta^{2} \frac{3-\kappa_{1}}{\kappa_{1}+1}\right)=0
\end{aligned}
$$

The roots of Eq. (10) can be expressed as given.

$$
\begin{aligned}
& n_{1}=-\frac{1}{2}\left(\beta+\sqrt{4 \xi^{2}+\beta^{2}-4 \xi \beta i \sqrt{\frac{3-\kappa}{\kappa+1}}}\right) \\
& n_{2}=-\frac{1}{2}\left(\beta-\sqrt{4 \xi^{2}+\beta^{2}-4 \xi \beta i \sqrt{\frac{3-\kappa}{\kappa+1}}}\right) \\
& n_{3}=-\frac{1}{2}\left(\beta+\sqrt{4 \xi^{2}+\beta^{2}+4 \xi \beta i \sqrt{\frac{3-\kappa}{\kappa+1}}}\right) \\
& n_{4}=-\frac{1}{2}\left(\beta-\sqrt{4 \xi^{2}+\beta^{2}+4 \xi \beta i \sqrt{\frac{3-\kappa}{\kappa+1}}}\right)
\end{aligned}
$$

Solving ordinary differential equation represented by (12), the displacement and stress components of the FG layer can be expressed as follows.

$$
\begin{aligned}
& u(x, y)=\int_{0}^{\infty} \sum_{k=1}^{4}\left(A_{k} e^{n_{k} y}\right) e^{-i \xi x} d \xi \\
& v(x, y)=\int_{0}^{\infty} \sum_{k=1}^{4}\left(m_{k} A_{k} e^{n_{k} y}\right) e^{-i \xi x} d \xi \\
& \sigma_{y}(x, y)=\frac{G(y)}{K-1} \int_{0}^{\infty} \sum_{k=1}^{4}\left(C_{k} A_{k} e^{n_{k} y}\right) e^{-i \xi x} d \xi
\end{aligned}
$$


$\tau_{x y}(x, y)=G(y) \int_{0}^{\infty} \sum_{k=1}^{4}\left(D_{k} A_{k} e^{n_{k} y}\right) e^{-i \xi x} d \xi$

In these components; $m_{k}, C_{k}$ and $D_{k}(k=1, \ldots, 4)$ are known functions whereas $A_{k}$ are unknown coefficient functions. Applying boundary conditions (4-7), unknown coefficients can be obtained in terms of unknown contact pressures, $p_{j}(j=1,2, \ldots, n)$, and contact distances, $b_{j L}$ and $b_{j R}$.

Using unused boundary condition given in Eq. (8), the solution of the problem can be converted into the solution of an integral equation system which consists of $n$ singular integral equations and unknown contact pressures and contact distances can be obtained using the solution method suggested in [36] from the solution of this system.

Since the problem involves arbitrary loading, the analytical solution of the problem should be carried out for each loading case. In order to achieve this, a computer program is coded such that it performs the analytical solution of the problem using suggested method and displays obtained results.

\section{Numerical results and discussion}

The height of the graded layer, $h$, is taken as 1 whereas the Poison's ratio of the graded layer is taken as 0,25 . Note that all quantities in the tables and figures are normalized. $G_{h}$ defined as the shear modulus at the top of the FG layer $(y=h)$.

$$
G_{h}=G_{0} e^{\beta h}
$$

As the first loading case, a symmetric problem from the literature [27] is chosen in order to compare the results. In this problem, the layer is subjected to a concentrated load, $P$, by means of a circular rigid block with radius, $R$, placed at the symmetry axis and is resting on a Winkler foundation. The contact distances occurs in $[-a, a]$ in [27] whereas the contact distances occurs in $\left[b_{1 L}, b_{1 R}\right]$ in this study. Since the problem is symmetric with respect to geometry as well as loading, comparing only $a$ and $b_{1 R}$ values can be sufficient.

Table 1 shows the comparison of $a$ and $b_{1 R}$ for various $G_{0} / G_{h}$ and $k / G_{h}$ ratios. It can be seen that increasing $G_{0} / G_{h}$ and $k / G_{h}$ results a reduction in contact distances. In addition, the written program produces almost same values in the literature.

The contact pressures for various $G_{0} / G_{h}$ ratios are given in Fig. 2. As it is seen from the figure, if the $G_{0} / G_{h}$ ratio increases, contact distances decreases. Moreover, contact pressures increases for decreasing contact distances. Maximum pressures occur under the middle of the circular block.

As the second loading case, a symmetric problem with two identical circular blocks is chosen. The blocks are placed such that the distances from the middle of the blocks to the $y$ axis is same and loaded with same concentrated loads. The first block is at the left whereas the second block is at the right.

Table 2 shows the contact distances for various block positions. As it can be seen from the table, since the problem is symmetric, obtained contact distances for one block are compatible with the contact distances of the other block. In addition, when blocks approach to each other, $b_{1 R}$ and $b_{2 L}$ values approach to the middle of the block whereas $b_{1 L}$ and $b_{2 R}$ values move away from the middle of the block.

The change in contact pressures under the left block and right block for various block positions is given in Fig. 3 and 4, respectively. It can be seen from the figures, maximum contact pressures increases if the block approaches to each other. Also, the pressures under the block are comparable because of symmetry. 


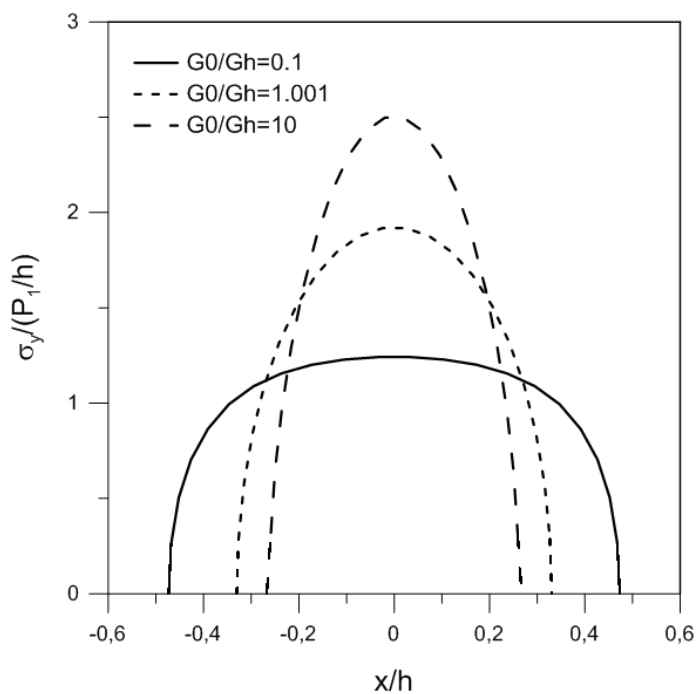

Fig. 2. Dimensionless contact pressures for various $G_{0} / G_{h}$ ratios in case of one block

Table 1. The comparison of half contact distances for various $G_{0} / G_{h}$ and $k / G_{h}$ ratios in case of one block $(R / h=100)$

\begin{tabular}{|c|c|c|c|c|c|c|}
\hline \multirow{2}{*}{$\frac{k}{G_{h}}$} & \multicolumn{2}{|c|}{$\frac{G_{0}}{G_{h}}=0.1$} & \multicolumn{2}{|c|}{$\frac{G_{0}}{G_{h}}=1.001$} & \multicolumn{2}{|c|}{$\frac{G_{0}}{G_{h}}=10$} \\
\hline & $\begin{array}{c}\text { Comez } \\
{[27]}\end{array}$ & $\begin{array}{c}\text { Present } \\
\text { study }\end{array}$ & $\begin{array}{c}\text { Comez } \\
{[27]} \\
\end{array}$ & $\begin{array}{c}\text { Present } \\
\text { study }\end{array}$ & $\begin{array}{c}\text { Comez } \\
{[27]}\end{array}$ & $\begin{array}{c}\text { Present } \\
\text { study }\end{array}$ \\
\hline 0.1 & 0.6220 & 0.6222 & 0.3725 & 0.3724 & 0.2779 & 0.2780 \\
\hline 1 & 0.4734 & 0.4732 & 0.3304 & 0.3304 & 0.2673 & 0.2674 \\
\hline 10 & 0.4452 & 0.4451 & 0.3151 & 0.3150 & 0.2617 & 0.2618 \\
\hline$\rightarrow \infty$ & 0.4415 & 0.4420 & 0.3116 & 0.3115 & 0.2594 & 0.2595 \\
\hline
\end{tabular}

Table 2. The contact distances for various block positions in case of two symmetrical blocks $\left(G_{0} / G_{h}=0.5\right.$, $\left.P_{1} / P_{2}=1, k / G_{h}=0.1, R_{1} / h=100\right)$

\begin{tabular}{ccccc}
\hline$|x / h|$ & $b_{1 L}$ & $b_{1 R}$ & $b_{2 L}$ & $b_{2 R}$ \\
\hline 5 & -5.240230 & -4.759518 & 4.759518 & 5.240230 \\
1 & -1.240149 & -0.761891 & 0.761891 & 1.240149 \\
0.5 & -0.758593 & -0.290634 & 0.290634 & 0.758593 \\
0.25 & -0.535542 & -0.083235 & 0.083235 & 0.535542 \\
\hline
\end{tabular}




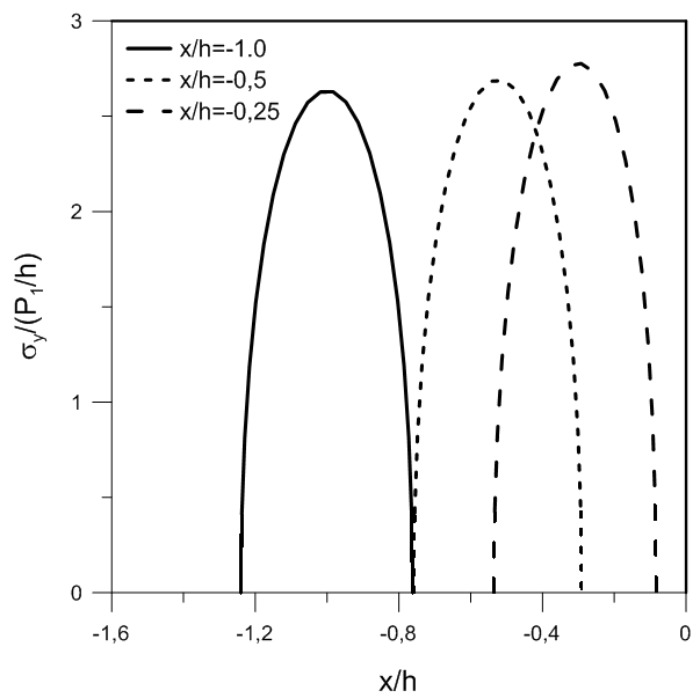

Fig. 3. Dimensionless contact pressures under the left block for various block positions in case of two symmetric blocks

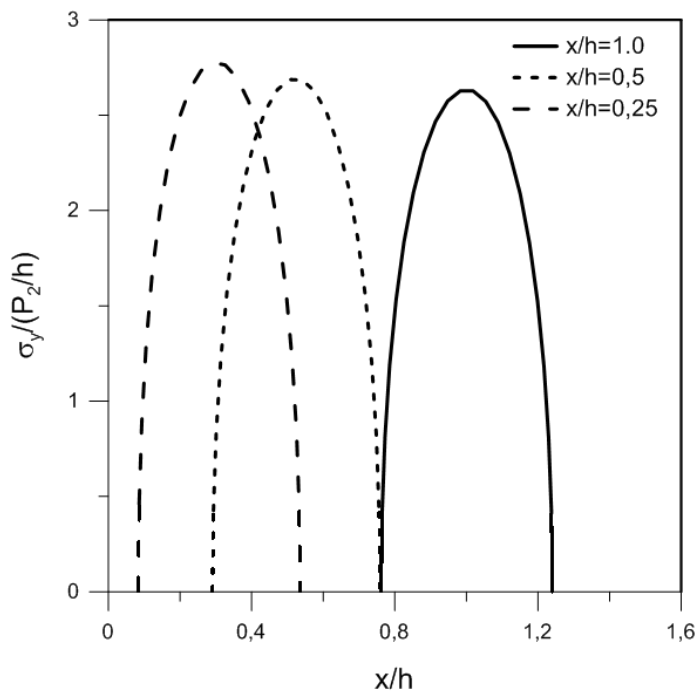

Fig. 4. Dimensionless contact pressures under the right block for various block positions in case of two symmetric blocks

As the last loading case, a problem with three blocks is chosen. The left and right blocks are cylindrical with similar or different radius. Whereas the block in the middle is flat. First, second and third blocks are at the left, in the middle and at the right, respectively.

The variation of contact distances for various block radius ratios is given in Table 3 . The radius of the right block is kept constant whereas the radius of the left block is changed. It can be seen from the table, the contact distances are not comparable between left and right blocks in case of asymmetric loading $\left(R_{1} / R_{3} \neq 1\right)$. In addition, the start and end of the contact distances goes to left and right, respectively, if $R_{1} / R_{3}$ increases. In other words, the contact distance increases because of the increase in the block radius. Although, the block radius of the right block is kept constant, there are small changes in the contact distances. Similar to left block, the start and end of the contact distances 
goes to left and right, respectively, and the contact distance increases if $R_{1} / R_{3}$ increases.

Figs. 5-7 show the change in the contact pressures for left block, right block and middle block, respectively, in case of various block radius ratios. As it is seen from the figures, if the radius of the block increases, the maximum contact pressure under the block decreases. Increasing the radius of the left block does not much affect in the contact pressure of the other two punches whether it is circular or flat and the contact pressure graphs for various block radius ratios are overlapped. Compared to circular blocks, the pressures under the flat block goes to infinite at the ends of the block.

Table 3. The contact distances for various block radius ratios in case of three asymmetrical blocks $\left(G_{0} / G_{h}=0.5\right.$, $\left.P_{1} / P_{2}=1, P_{1} / P_{3}=1, k / G_{h}=0.1, R_{3} / h=100\right)$

\begin{tabular}{ccccc}
\hline$R_{1} / R_{3}$ & $b_{1 L}$ & $b_{1 R}$ & $b_{3 L}$ & $b_{3 R}$ \\
\hline 0,25 & -2.117883 & -1.884599 & 1.766879 & 2.243265 \\
0,5 & -2.169165 & -1.835812 & 1.766802 & 2.243333 \\
1,0 & -2.243361 & -1.766792 & 1.766792 & 2.243361 \\
2,0 & -2.350717 & -1.671286 & 1.766743 & 2.243644 \\
\hline
\end{tabular}

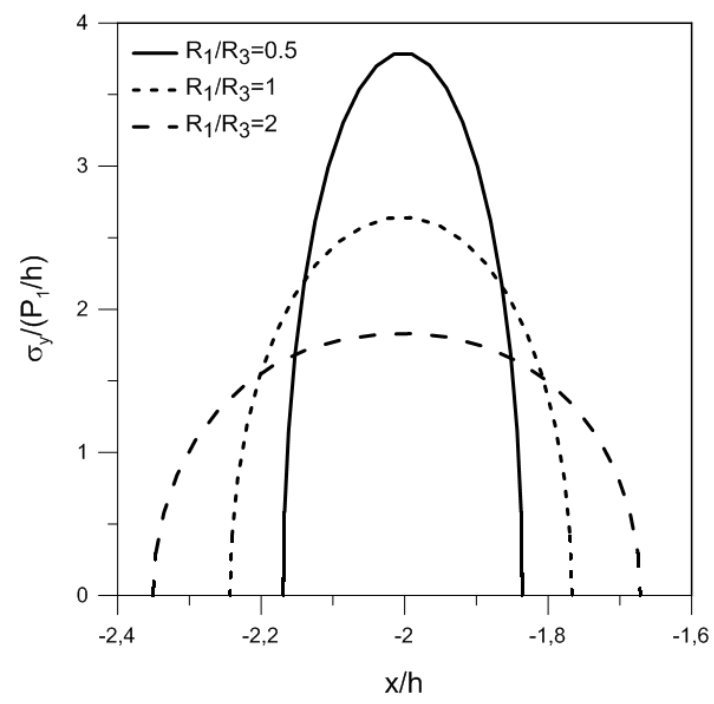

Fig. 5. Dimensionless contact pressures under the left block for various block radius ratios in case of three asymmetric blocks 


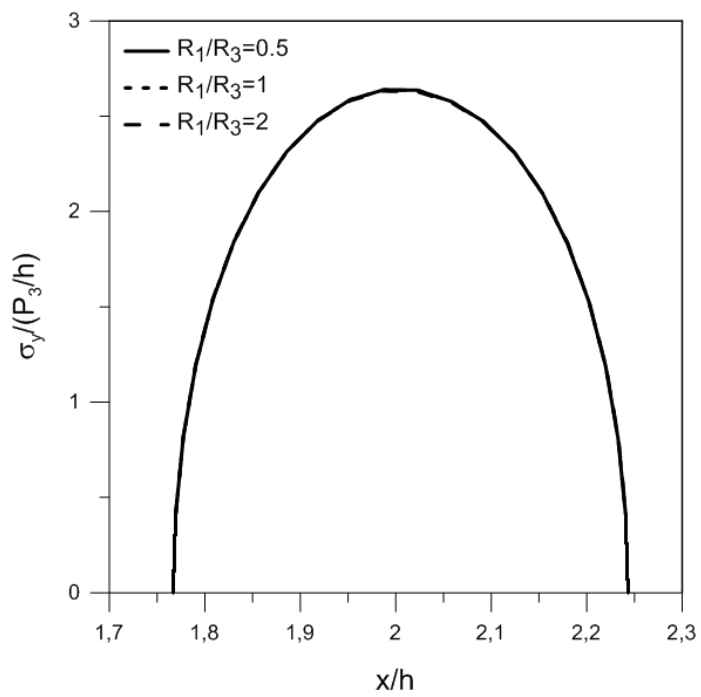

Fig. 6. Dimensionless contact pressures under the right block for various block radius ratios in case of three asymmetric blocks

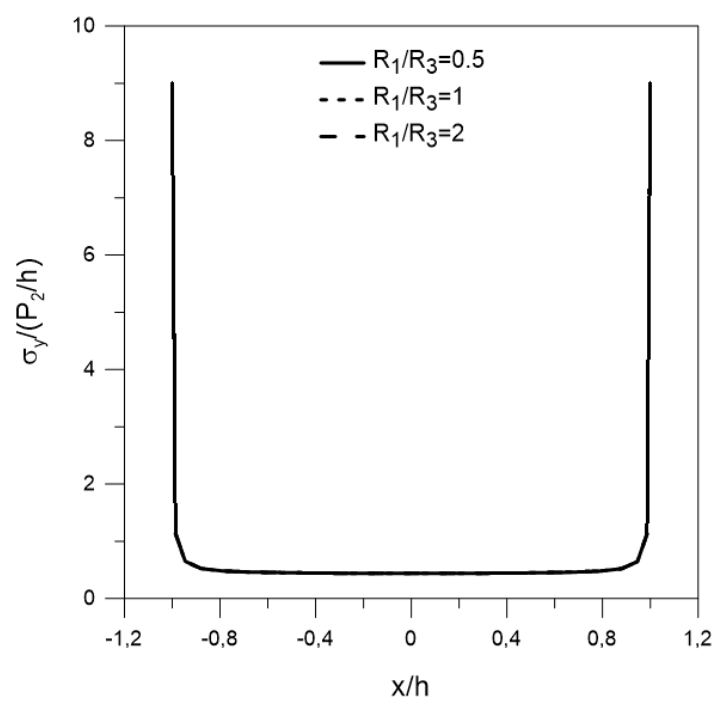

Fig. 7. Dimensionless contact pressures under the middle block for various block radius ratios in case of three asymmetric blocks

\section{References}

[1] Garrido JA, Lorenzana A (1998) Receding contact problem involving large displacements using the BEM. Engineering Analysis with Boundary Elements 21(4): 295-303.

[2] Adibelli H, Comez I, Erdol R (2013) Receding contact problem for a coated layer and a halfplane loaded by a rigid cylindrical stamp. Archives of Mechanics 65(3): 219-236.
[3] Keer LM, Dundurs J, Tsai KC (1972) Problems involving a receding contact between a layer and a half Space. Journal of Applied Mechanics, Transactions ASME 39(4): 1115-1120.

[4] Erdogan F, Ratwani M (1974) The contract problem for an elastic layer supported by two elastic quarter planes. Journal of Applied Mechanics 41(3): 673-678. 
[5] Civelek MB, Erdogan F (1974) The axisymmetric double contact problem for a frictionless elastic layer. International Journal of Solids Structures 10(6): 639-659.

[6] Akavc1 S. The frictionless contact problem of an elastic layer supported by two elastic quarter planes. PhD Thesis, Çukurova University, 1999.

[7] Comez I, Birinci A, Erdol R (2004) Double receding contact problem for a rigid stamp and two elastic layers. European Journal of Mechanics-A/Solids 23(2): 301-309.

[8] Kahya V, Ozsahin TS, Birinci A, Erdol R (2007) A receding contact problem for an anisotropic elastic medium consisting of a layer and a half plane. International Journal Solids Structures 44(17): 5695-5710.

[9] Yaylaci M, Birinci A (2013) The receding contact problem of two elastic layers supported by two elastic quarter planes. Structural Engineering and Mechanics 48(2): 241-255.

[10] Oner E, Yaylaci M, Birinci A (2014) Solution of a receding contact problem using an analytical method and a finite element method. Journal of Mechanics of Materials and Structures 9(3): 333345.

[11] Karabulut PM, Adiyaman G, Birinci A (2017) A receding contact problem of a layer resting on a half plane. Structural Engineering and Mechanics 64(4): 505-513.

[12] Kieback B, Neubrand A, Riedel H (2003) Processing techniques for functionally graded materials. Materials Science and Engineering: A 362(1): 81-106.

[13] Jin ZH, Batra RC (1996) Stress intensity relaxation at the tip of an edge crack in a functionally graded material subjected to a thermal shock. J Thermal Stresses 19(4): $317-$ 339.

[14] Shen HS. Functionally Graded Materials: Nonlinear Analysis of Plates and Shells. CRC Press, 2009

[15] Sofiyev AH (2011) Thermal buckling of FGM shells resting on a two-parameter elastic foundation. Thin-Walled Structures 49(10): 1304-1311.

[16] Bagherizadeh E, Kiani Y, Eslami MR (2012) Thermal buckling of functionally graded material cylindrical shells on elastic foundation. The American Institute of Aeronautics and Astronautics Journal 50(2): 500-503.
[17] Tornabene F, Fantuzzi N, Viola E, Batra RC (2015) Stress and strain recovery for functionally graded free-form and doubly-curved sandwich shells using higher-order equivalent single layer theory. Composite Structures 119: 67-89.

[18] El-Borgi S, Abdelmoula R, Keer L (2006) A receding contact plane problem between a functionally graded layer and a homogeneous substrate. International Journal of Solids and Structures 43(3-4): 658-674.

[19] Ke LL, Wang YS (2007) Two-dimensional sliding frictional contact of functionally graded materials. European Journal of MechanicsA/Solids 26(1): 171-188.

[20] Yang J, Ke LL (2008) Two-dimensional contact problem for a coating-graded layer-substrate structure under a rigid cylindrical punch. International Journal of Mechanical Sciences 50(6): 985-994.

[21] Barik SP, Kanoria M, Chaudhuri PK (2009) Frictionless contact of a functionally graded halfspace and a rigid base with an axially symmetric recess. Journal of Mechanics 25(1): 9-18.

[22] Ke LL, Yang J, Kitipornchai S, Wang YS (2008) Electro-mechanical frictionless contact behavior of a functionally graded piezoelectric layered half-plane under a rigid punch. International Journal of Solids and Structures 45(11-12): 33133333.

[23] Dag S, Guler MA, Yidirim B, Ozatag AC (2009) Sliding frictional contact between a rigid punch and a laterally graded elastic medium. International Journal of Solids and Structures 46(22-23): 4038-4053.

[24] Rhimi M, El-Borgi S, Ben Said W, Ben Jemaa F (2009) A receding contact axisymmetric problem between a functionally graded layer and a homogeneous substrate. International Journal of Solids and Structures 46(20): 3633-3642.

[25] Rhimi M, El-Borgi S, Lajnef N (2011) A double receding contact axisymmetric problem between a functionally graded layer and a homogeneous substrate. Mechanics of Materials 43(12): 787798.

[26] Chen PJ, Chen SH (2012) Contact behaviors of a rigid punch and a homogeneous half-space coated with a graded layer. Acta Mechanica 223(3): 563-577.

[27] Comez I (2013) Contact problem of a functionally graded layer resting on a Winkler 
foundation. Acta Mechanica 224(11): 2833 2843.

[28] El-Borgi S, Usman S, Guler MA (2014) A frictional receding contact plane problem between a functionally graded layer and a homogeneous substrate. International Journal of Solids and Structures 51(25-26): 4462-4476.

[29] Adiyaman G, Birinci A, Oner E, Yaylaci M (2016) A receding contact problem between a functionally graded layer and two homogeneous quarter planes. Acta Mechanica 227(6): 17531766.

[30] Yan J, Li X (2015) Double receding contact plane problem between a functionally graded layer and an elastic layer. European Journal of MechanicsA/Solids 53: 143-150.

[31] Liu TJ, Xing YM, Wang YS (2016) The axisymmetric contact problem of a coating/substrate system with a graded interfacial layer under a rigid spherical punch. Mathematics and Mechanics of Solids 21(3): 383-399.
[32] Turan M, Adiyaman G, Kahya V, Birinci A (2016) Axisymmetric analysis of a functionally graded layer resting on elastic substrate. Structural Engineering and Mechanics 58(3): 423-442.

[33] Yan J, Mi C (2017) On the receding contact between an inhomogeneously coated elastic layer and a homogeneous half-plane. Mechanics of Materials 112: 18-27.

[34] Yan J, Mi C (2017) Double contact analysis of multilayered elastic structures involving functionally graded materials. Archives of Mechanics 69(3): 199-221.

[35] Dempsey JP, Zhao ZG, Minnetyan L, Li H (1990) Plane contact of an elastic layer supported by a Winkler foundation. Journal of Applied Mechanics, Transactions ASME 57(4): 974-980.

[36] Erdogan F, Gupta, GD (1972) Numerical solution of singular integral-equations. Quarterly of Applied Mathematics 29(4): 525. 